\title{
Defining and controlling double quantum dots in single-walled carbon nanotubes
}

\author{
MR Gräber, M Weiss, and C Schönenberger \\ Institut für Physik, Universität Basel, Klingelbergstrasse 82, CH-4056 Basel, \\ Switzerland \\ E-mail: christian.schoenenberger@unibas.ch
}

\begin{abstract}
We report the experimental realization of double quantum dots in singlewalled carbon nanotubes. The device consists of a nanotube with source and drain contact, and three additional top-gate electrodes in between. We show that, by energizing these top-gates, it is possible to locally gate a nanotube, to create a barrier, or to tune the chemical potential of a part of the nanotube. At low temperatures we find (for three different devices) that in certain ranges of top-gate voltages our device acts as a double quantum dot, evidenced by the typical honeycomb charge stability pattern.
\end{abstract}




\section{Introduction}

Since their discovery in 1991 [1] carbon nanotubes have, due to their unique mechanical and electronic properties, been subject of a tremendous scientific and technological interest. In the field of mesoscopic physics, carbon nanotubes offer an easily accessible experimental platform for studying the physics of the text book example of a particle trapped inside a box, a so-called quantum dot or artificial atom [2, 3. Single quantum dots can simply be realized by contacting a nanotube with two metallic contacts (normally made of Palladium); the contacts between the nanotube and the metallic leads usually act as tunnel barriers, characterized by the nanotube-lead tunneling rate $\Gamma$ and a capacitance $C$. The energy scales for nanotube quantum dots are given by a typical single-electron charging energy $U_{C} \approx 3 \mathrm{meV} \approx 30 \mathrm{~K}$ and a quantummechanical level spacing $\delta E=\frac{h v_{F}}{2 L}$, where $h$ is Planck's constant. Using the Fermi velocity $v_{F}=8 \times 10^{5} \mathrm{~m} / \mathrm{s}$ and an effective nanotube length $L=1 \mu \mathrm{m}$ the level spacing amounts to $\delta E \approx 2 \mathrm{meV}$.

However, the standard approach for manufacturing quantum dot devices has relied on structures in GaAs-based 2-dimensional electron gases (2-DEG), which can be defined using etching and gating techniques. The main advantage of this system is the high degree of control over the quantum dot properties, which has been achieved over the last years. These quantum dots allow for a precise tuning of the coupling to the leads by energizing locally acting gate electrodes, see e.g. 4] and references therein. Additionally, center gates can be used in order to define double quantum dot structures with a tunable inter-dot coupling. This tunability is an essential ingredient for further experiments exploring the nature of electronic states in quantum dots - or, even more ambitious, for realizing quantum electronic devices such as spin- or charge-based quantum bits [5, 6, 7]. Whereas this high degree of control has been lacking in nanotube-based quantum dots so far, using carbon nanotubes offers fascinating opportunities. For example, new physical phenomena such as superconducting correlations or spin injection into quantum dots can be studied in carbon nanotube quantum dots [8, 9]. In contrast to carbon nanotubes, up to now it has not been possible to attach ferromagnetic and superconducting to GaAs-based quantum dots. Moreover, the influence of the surrounding nuclear spins is expected to limit electron spin dephasing times in GaAs (double) quantum dots [10]. In carbon nanotubes, on the other hand, nuclear spins are predominantly absent and hyperfine interactions thus strongly reduced. The question, to which degree carbon nanotube quantum dots can be tuned using locally acting gate electrodes is therefore an important issue to address. In this article we describe a technique of implementing local top-gate electrodes onto a single-walled carbon nanotube (SWNT). After characterizing the functionality of the top-gates we will then make use of them in order to define and control double quantum dots inside SWNTs. 

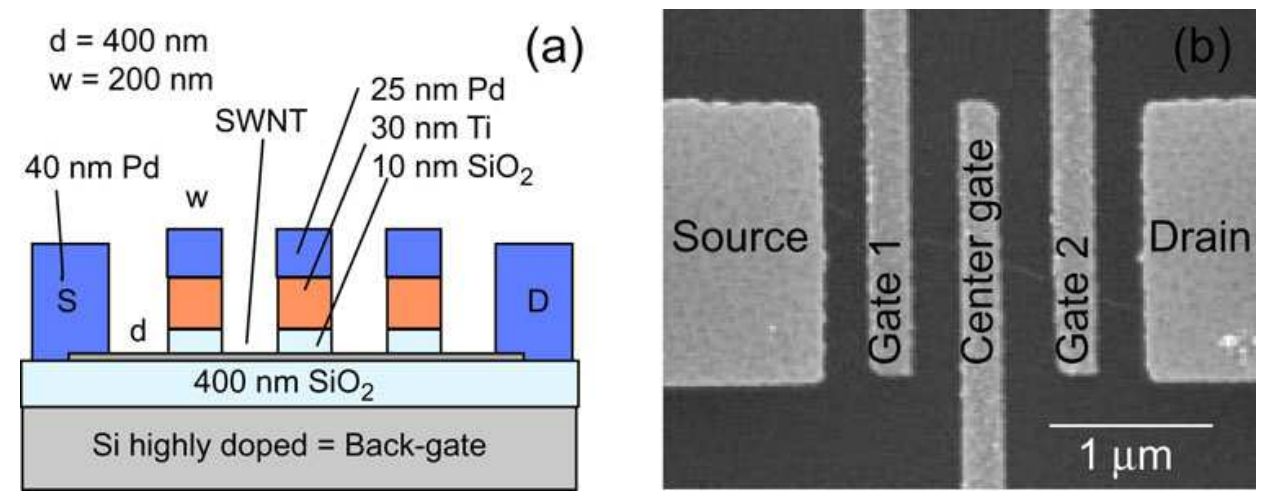

Figure 1. (a) Side-view schematic of a SWNT device with three top-gates. (b) Scanning electron micrograph of the device. Gates are labelled gate 1, center gate, and gate 2 (from source to drain).

\section{Local gating of carbon nanotubes}

\subsection{Strategies for gating nanotube quantum dots}

With the nanotube lying on an oxidized Si-substrate, a natural way of gating this single quantum dot is to apply a voltage to the doped Si-substrate. The Si then acts as a back-gate globally affecting the whole quantum dot. In order to create multiple dots in such a device and control them independently, however, one will need to find a way of locally gating a nanotube. By using such local gates, one can either create a barrier, or simply shift the chemical potential within a small part of the nanotube. In the following, we will briefly review two different strategies of local gating of nanotubes that have been reported in the literature, and will describe in detail the technique that has been developed in our lab. At the end of this section measurements of electrical transport through nanotube devices with local gates will be presented.

In order to fabricate gate electrodes locally acting on a nanotube, side-gates represent a straightforward option [11]. Besides source and drain contact, additional electrodes are patterned in the vicinity of the nanotube. The advantage of this technique is that contacts and side-gates can be fabricated within the same processing step. It is, however, difficult to get the side-gates as close to the nanotube as possible, yet not contacting it electrically. Thus, typically side-gates are spaced by approximately $100 \mathrm{~nm}$ from the SWNT, making the gating less efficient and their action less local.

More efficient are gates made by directly evaporating the gate electrode on top of the nanotube, with a thin gate oxide underneath. These so-called topgates are spaced from the SWNT only by the thickness of the gate oxide $(\approx 1-10 \mathrm{~nm})$, making them act more efficiently and (depending on their width) more locally as compared to side-gates. Despite the fact that there are drawbacks of this method as well (additional processing steps, nanotube properties may be modified underneath the top-gates), top-gates are the most promising approach for creating local barriers in SWNT. Therefore, we have developed a reliable method for fabricating top-gate electrodes in our laboratory, which 
we will now discuss in more detail.

\subsection{Experimental}

SWNTs were grown on a degenerately doped $\mathrm{Si} / \mathrm{SiO}_{2}$ substrate by means of chemical vapor deposition (CVD). Details of the CVD process can be found elsewhere [12. After the initial preparation of $\mathrm{SiO}_{2} / \mathrm{Ti} / \mathrm{Au}$ bond pads and alignment markers, SWNTs were then localized with a scanning electron microscope (SEM). In the following step the gate electrodes were defined by e-beam-lithography. Electron-gun-evaporation of $\mathrm{SiO}_{2}$ as gate-oxide, Ti as gate-metal, and Pd serving as anti-oxidant cover layer followed. The gate-oxide film thickness was chosen to be $10 \mathrm{~nm}$, the Ti film thickness $30 \mathrm{~nm}$, and that of the Pd layer $25 \mathrm{~nm}$. The materials were evaporated at a pressure of $\approx 10^{-7}$ mbar. In a final lithography and evaporation step the source and drain electrodes of the nanotube, consisting of $40 \mathrm{~nm} \mathrm{Pd}$, were defined. The evaporation conditions were the same as described above, except the substrate was kept at a constant temperature of $\approx 0^{\circ} \mathrm{C}$ by cooling the sample holder inside the evaporation chamber. This cooling helps to reduce outgasing of materials inside the vacuum chamber due to heating during the evaporation. After lift-off of the remaining PMMA, the samples were glued into a 20-lead chip carrier and bonded. Figures 1(a) and (b) show a side-view schematic and a scanning electron micrograph of a typical SWNT device with three top-gates in addition to the source and drain electrode. The spacing between source and drain electrode amounts to $2.2 \mu \mathrm{m}$, and the width of the gates was chosen to be $200 \mathrm{~nm}$. The back-gate oxide has a commonly used thickness of $400 \mathrm{~nm}$.

\subsection{Effect of local gate electrodes at $300 \mathrm{~K}$ and at $4.2 \mathrm{~K}$}

In Fig. 2(a) the linear conductance versus gate voltage of a device with three topgate electrodes is plotted. The gate-dependence identifies the semiconducting nature of the SWNT. At a voltage of roughly $0.6 \mathrm{~V}$ applied to either of the three topgates the conductance through the device is suppressed indicating that the chemical potential is shifted locally into the semiconducting gap of the SWNT. After a decrease of conductance for increasing gate voltage, the conductance rises again for more positive gate voltages. This behavior is explained by the band diagram sketched in the inset of Fig. 2(a). Intrinsically the tube is p-doped and the chemical potential $\mu$ resides in the valence band (i). For increasing voltage at the top-gate the potential landscape is changed locally, making $\mu$ lie within the energy gap below the gate (ii). In this scenario the conductance through the nanotube reaches its minimum. With this technique it should thus be possible to create local barriers inside a carbon nanotube, allowing one to create artificial potential landscapes. If the gate voltage is increased even more, the lower edge of the conduction band will eventually reach the upper edge of the valence band (iii). Now thermally activated band-to-band processes indicated by the green arrows are possible and the conductance increases again. We have observed such behavior only at $300 \mathrm{~K}$ indicating the large activation barriers involved in these band- 

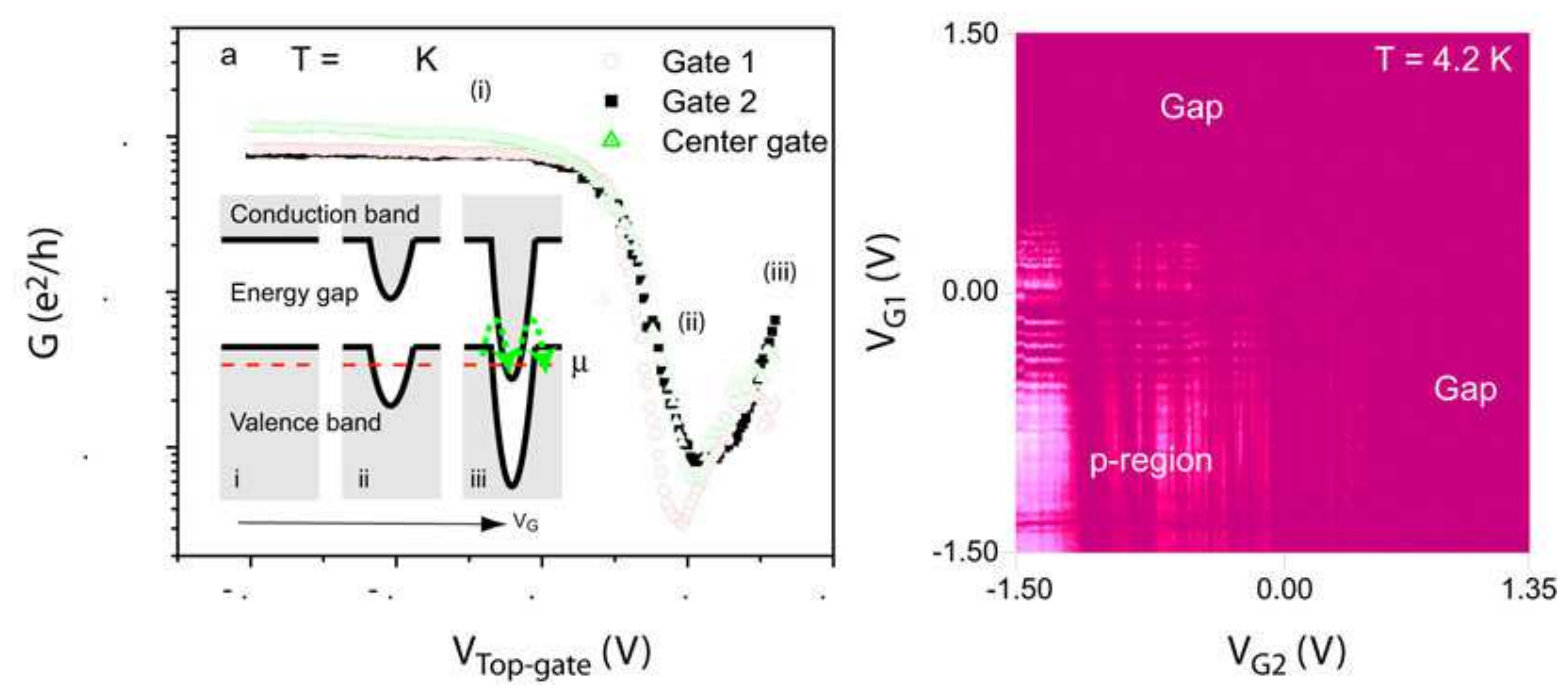

Figure 2. (a) Linear conductance $G$ on a logarithmic scale for a device with three top-gate electrodes (oxide thickness $10 \mathrm{~nm}$ ) versus top-gate-voltage at $\mathrm{T}=300 \mathrm{~K}$. The gates non swept are connected to ground potential. Inset: (i) - (iii) illustrate the band structure for increasing top-gate voltage. (b) Colorscale plot (dark $=0$, bright $=0.008 \mathrm{e}^{2} / \mathrm{h}$ ) of the conductance versus gate 1 and gate 2 for constant center-gate voltage at $4.2 \mathrm{~K}$.

to-band charge transfer processes. Band-to-band charge transfer processes have also been reported in Ref. [13.

In Fig. 2(b) the linear differential conductance at $4.2 \mathrm{~K}$ is plotted on a colorscale (bright $=$ more conductive) versus voltages applied at the top-gates 1 and 2 for a constant center-gate voltage of $V_{C}=-1 \mathrm{~V}$. At voltages of around $0.5 \mathrm{~V}$ applied to either of the top-gates the chemical potential is shifted into the energy gap of the nanotube and electrical transport is suppressed. For lower top-gate voltages, sweeping gate 1 and gate 2 leads to pronounced oscillations of the conductance due to single-electron charging and finite-size effects of the nanotube, which are accessible at low temperatures.

\section{Nanotube double quantum dots}

\subsection{Previous work}

Recently, in the field of double quantum dots in carbon nanotubes an enormous progress has been achieved. In 2004 Mason et al. first demonstrated the local gate control of an intrinsic double quantum dot inside a carbon nanotube [15. This work was then extended by the same group in Ref. [16], where a tunable mutual capacitance was demonstrated. In a recent work, Sapmaz et al. could observe electronic transport through excited states as seen in finite-bias triangles in a SWNT double dot [17. In Ref. [18] molecular eigenstates of a strongly coupled carbon nanotube double quantum dot were observed and analyzed. 


\subsection{Experimental data}

In this section we will show that it is possible to reliably define clean double quantum dots in SWNTs by using top-gate electrodes. We focus on three devices labelled A,B,C with three top-gates each. Samples A and B were fabricated according to Fig. 1(a). In the case of device $\mathrm{C}$ the source -drain spacing was reduced to $1.4 \mu \mathrm{m}$ and the top-gate width to $100 \mathrm{~nm}$. Whereas devices $\mathrm{A}$ and $\mathrm{B}$ are based on a semiconducting SWNT (operated in the hole regime), device $\mathrm{C}$ is metallic. In Fig. 33(a)(c) the differential conductance versus voltages applied at two top-gates is plotted on a colorscale (bright $=$ more conductive). For devices $\mathrm{A}$ and $\mathrm{C}$ the center gate has been set to a constant value of $-0.1 \mathrm{~V}$ and $0 \mathrm{~V}$, respectively, and gate 1 and gate 2 are swept. In case of device B, center gate and gate 1 are swept, while gate 2 was kept at a constant voltage of $V_{G 2}=-0.1 \mathrm{~V}$. The visible high-conductance ridges as observed for all three devices define a charge-stability map that is shaped like a honeycomb. This honeycomb pattern is characteristic of a double quantum dot. Within each cell, the number of holes $(\mathrm{n}, \mathrm{m})$ on the two dots is constant. Energizing gate 1 (2) to more negative voltages successively fills holes into dot 1 (2), whereas a more positive voltage pushes holes out of the dot. The fact that all three devices can be tuned to exhibit a honeycomb pattern shows that the double quantum dots are indeed defined by the local gates and not intrinsic to the nanotube. Common to all three devices is that the applied gate voltages are close to $0 \mathrm{~V}$, i.e. far off the pinch-off voltage. In such a regime, we expect a smooth modulation of the electronic potential rather than sharp and steep barriers.

The honeycomb charge stability map allows for a quantitative determination of the double dot capacitances as defined in the electrostatic double dot model in Fig. 3)(e), following the work of van der Wiel et al. 14. As an example we will determine the capacitances of the double dot defined in device C, see Fig. 31(c). From the dimensions of the honeycomb cell one can extract the gate capacitances:

$$
C_{G 1 / 2}=|e| / \Delta V_{G 1 / 2},
$$

yielding $C_{G 1} \approx 30 \mathrm{aF}$ and $C_{G 2} \approx 25 \mathrm{aF}$. Of particular importance are the points where three charge states are degenerate, so-called triple points. Two such points are marked by dashed circles in Fig. 3(b) for clarity. When applying a finite bias voltage, the triple points transform into triangles, in which transport is enabled. Fig. 3(d) shows the triple point region within the dashed box of Fig. 3(c) at an applied source-drain voltage of $V_{s d}=500 \mu V$. From the dimensions of these triangles $\delta V_{G 1(G 2)}$ and

$$
C_{G 1(G 2)} / C_{1(2)}=\left|V_{s d}\right| / \delta V_{G 1(G 2)},
$$

we obtain the total capacitance $C_{1}=C_{s}+C_{G 1}+C_{m} \approx 60 \mathrm{aF}$ and $C_{2}=C_{d}+C_{G 2}+C_{m} \approx$ 75 aF of dot 1 and dot 2, respectively. Here $C_{m}$ denotes the mutual capacitance and $C_{s(d)}$ the capacitance of the tunnel barrier to source (drain). In a purely electrostatic model the mutual capacitance can be evaluated from the spacing of two adjacent triple points. This spacing, however, is influenced by the tunnel coupling $t$ in between the two dots as well. This quantum mechanical effect leads to a level anti-crossing, resulting 

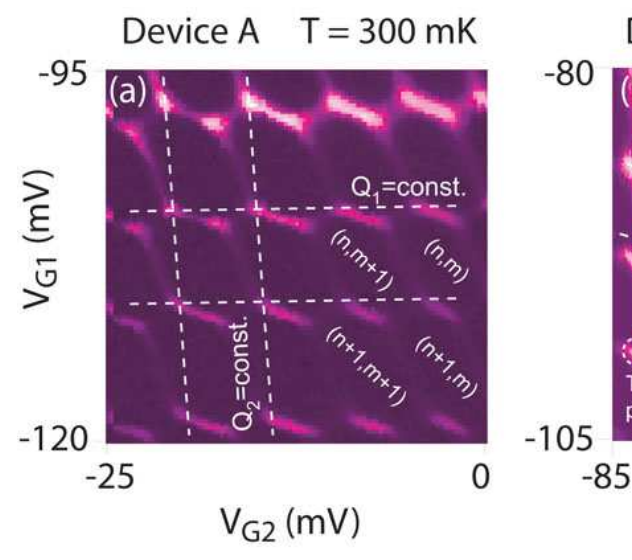

Device B $\quad T=500 \mathrm{mK}$

(e)

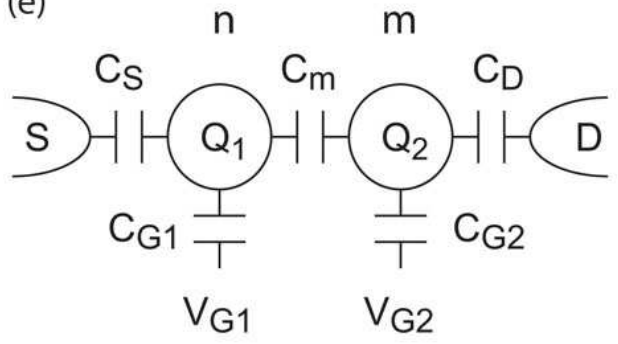

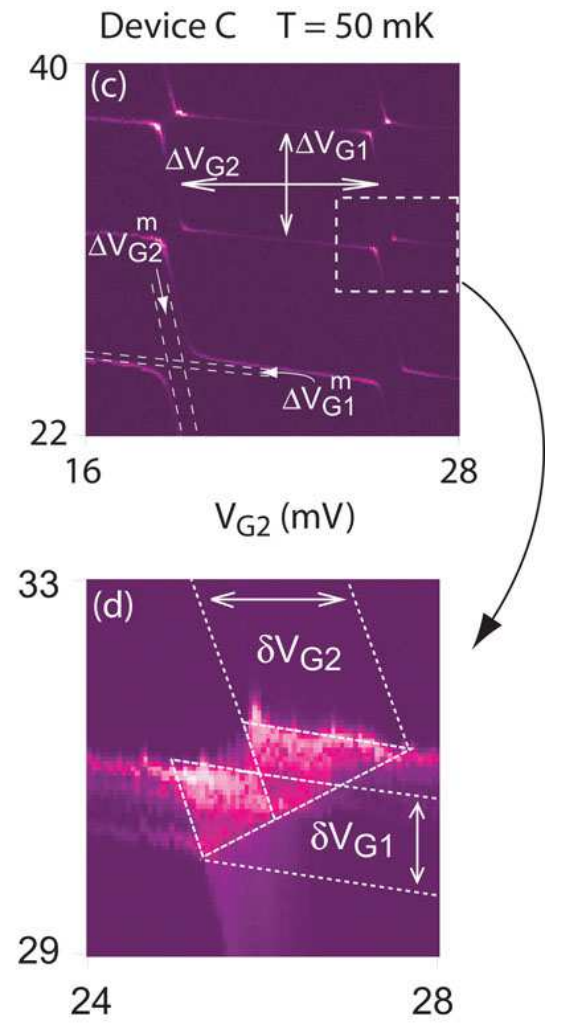

24

Figure 3. (a) Colorscale plot of the conductance versus top-gate voltages at $300 \mathrm{mK}$ for device A. Bright corresponds to $0.4 \mathrm{e}^{2} / \mathrm{h}$. The obtained honeycomb pattern is the charge stability map of a double quantum dot. (b) Same for device B at $500 \mathrm{mK}$, bright corresponds to $0.08 \mathrm{e}^{2} / \mathrm{h}$. (c) Same for device $\mathrm{C}$ at $50 \mathrm{mK}$, bright corresponds to $0.035 \mathrm{e}^{2} / \mathrm{h}$. (d) Zoom into the triple point region marked by the dashed box in (c) at a bias voltage of $V_{s d}=500 \mu \mathrm{V}$. (e) Capacitive model of a double quantum dot.

in curved wings in the vicinity of the triple points. A rough estimate of the mutual capacitance, however, can be achieved by drawing the asymptotes to the curved borders of the honeycomb, see the bottom left triple point region of Fig. B(c). From the vertical (horizontal) distance $\Delta V_{G 1}^{m}\left(\Delta V_{G 2}^{m}\right)$ it is then possible to extract $C_{m}$ by using

$$
\Delta V_{G 1,2}^{m}=|e| C_{m} / C_{G 1,2} C_{2,1} \text {. }
$$

We obtain a mutual capacitance of $C_{m} \approx 5 \mathrm{aF}$. Additionally, analyzing the curvature of the honeycomb borders allows one to precisely evaluate the tunnel coupling $t$. For a detailed description on this we refer to Ref. [18, where it was found that $t$ can exceed the electrostatic nearest-neighbor interaction by as much as an order of magnitude. This fact reflects the one-dimensional geometry of a nanotube; electrostatic interactions are reduced due to the large separation of the 'center of mass' of the charges (while still allowing a significant overlap of the wavefunctions). 


\subsection{Where exactly are the two dots?}

So far we have seen that it is possible to reliably define and control double quantum dots in SWNTs - the question where precisely the two dots are located, however, has not been addressed yet. As we will point out, from Fig. 3(a) and (b) it follows that the dots are separated by the center gate electrode. Recall that devices A and B are identical except that for device B the center gate (instead of gate 2) is used to control dot 2. The dashed lines in Fig. 3(a) and (b) connect triple points corresponding to a constant charge $Q_{1(2)}=$ const., residing on dot 1(2). A non-zero slope of these lines indicates a cross capacitance, i.e. the gate controlling one of the two dots also affects the chemical potential of the other. A non-zero slope is observed for the $Q_{1}=$ const.-lines in (b). Hence, the center gate affects both dot 1 and dot 2. On the other hand, this is not the case for gate 1 or gate 2 in Fig. 3(a) and (b). Such behavior can be explained assuming that the two dots are separated by the center gate, screening the cross-action of gates 1 and 2. The center gate, however, located in between the dots and creating a barrier, is not screened and thus acts on the two dots. If the center gate is capable of creating a tunnel barrier inside the nanotube, so will be gate 1 and gate 2 as well. Also recall that the voltages applied to the top-gates are all within the same range, $V_{\text {Top-gate }} \approx 0 \mathrm{~V}$. Consequently dot 1 is located between gate 1 and center gate, whereas dot 2 extends from the center gate to gate 2 . The scenario suggested implies that the part of the SWNT between gate 1(2) and source (drain) electrode has an effectively energy-independent transmission. In fact this assumption is quite reasonable, taking into account the high quality of Pd-nanotube electrical contacts [19]. Very transparent contacts $(\Gamma \approx \delta E)$ lead to a constant, or at least only slightly modulated transmission. Transport through our device will be dominated by the bottleneck in transmission - the gate-defined double quantum dot.

\section{Conclusions}

In this article we have presented a reliable approach to define and control double quantum dots in SWNTs by using locally acting top-gate electrodes. That the double quantum dots are not intrinsic to the carbon nanotubes is confirmed by the presented measurements of honeycomb patterns for three different devices. Furthermore, using an electrostatic model, we have been able to characterize the double-dot system quantitatively by extracting its capacitances. Despite these encouraging results, further research is necessary. Challenges to master include the gate-control of the quantummechanical tunnel coupling of the two quantum dots and the access to regimes of only a few charge carriers per dot. Carbon nanotubes may, due to their unique properties and their experimental ease, then play an important role in future information technology. 


\section{Acknowledgements}

For theoretical support we are gratefully indebted to W.A. Coish and D. Loss. We acknowledge experimental contributions by J. Furer, C. Hoffmann, and J. Gobrecht for oxidized Si substrates. Financial support from the Swiss NFS, the NCCR on Nanoscience, and the 'C. und H. Dreyfus Stipendium' (MRG) is greatly appreciated.

\section{References}

[1] Iijima S, Nature 56354 (1991).

[2] Kastner M, Physics Today 2446 (1993).

[3] Bockrath M, Cobden DH, McEuen PL, Chopra NG, Zettl A, Thess A, and Smalley RE, Science 1922275 (1997).

[4] Kouwenhoven LP, Marcus CM, McEuen PL, Tarucha S, Westervelt RM, and Wingreen NS, in Proceedings of the Advanced Study Institute on Mesoscopic Electron Transport, edited by Sohn LL, Kouwenhoven LP, Schön G, Kluwer (1997).

[5] Loss D and DiVincenzo DP, Phys. Rev. A 12057 (1998).

[6] Shnirman A and Schön G, Phys. Rev. B 1540057 (1998).

[7] Burkard G, Loss D, and DiVincenzo DP, Phys. Rev. B 207059 (1999).

[8] Buitelaar MR, Nussbaumer T, and Schönenberger C, Phys. Rev. Lett. 89256801 (2002).

[9] Sahoo S, Kontos T, Furer J, Hoffmann C, Gräber MR, Cottet A, and Schönenberger C, Nature Physics 199 (2005).

[10] Koppens FHL, Folk JA, Elzerman JM, Hanson R, Willems van Beveren LH, Vink IT, Tranitz HP, Wegscheider W, Kouwenhoven LP, Vandersypen LMK; Science 309, 1346 (2005).

[11] Biercuk MJ, Mason N, Martin J, Yacoby A, and Marcus CM, Phys. Rev. Lett. 02680194 (2005).

[12] Furer J, PhD Thesis, University of Basel (2005).

[13] Appenzeller J, Lin YM, Knoch J, and Avouris Ph, Phys. Rev. Lett. 93, 196805 (2004).

[14] van der Wiel WG, de Francheschi S, Elzermann JM, Fujisawa T, Tarucha S and Kouwenhoven LP, Rev. Mod. Phys. 75 (2003).

[15] Mason N, Biercuk MJ, and Marcus CM, Science 303, 655 (2004).

[16] Biercuk MJ, Garaj S, Mason N, Chow JM, and Marcus CM, Nano Letters, 5, 1267 (2005).

[17] Sapmaz S, Meyer C, Beliczynski P, Jarillo-Herrero P, and Kouwenhoven LP, to be published in Nano Letters (2006).

[18] Gräber MR, Coish WA, Hoffmann C, Weiss M, Furer J, Oberholzer S, Loss D, and Schönenberger C, cond-mat 0603367 (2006).

[19] Javey A, Guo J, Wang Q, Lundstrom M, and Dai H, Nature 654424 (2003). 